\title{
Effects of Intravenous Magnesium Sulfate on the Prevention of Pain Following Injection of Microemulsion Propofol
}

\author{
Se Jin Lee, Soon Im Kim, Sun Young Park, Mun-Gyu Kim, Ho Bum Cho, Yoo Mi Han \\ Department of Anesthesiology and Pain Medicine, Soonchunhyang University Seoul Hospital, Seoul, Korea
}

\begin{abstract}
Objective: The aim of this study was to investigate the effects of intravenous magnesium sulfate on the prevention of pain during the injection of microemulsion propofol. Magnesium is a known calcium channel blocker and a physiological N-methyl-D-aspartate receptor antagonist.

Methods: American Society of Anesthesiologists I and II adults $(n=114)$ undergoing general anesthesia for surgery were randomly assigned into two groups ( $n=57$ per group). This study was designed in prospective and double-blind manner. Patients in the LM group $(n=57)$ received pretreatment with $2 \%$ lidocaine $(40 \mathrm{mg})$ and magnesium sulfate $10 \mathrm{mg} / \mathrm{kg}$, while patients in group L $(n=57)$ received pretreatment with $2 \%$ lidocaine $(40 \mathrm{mg})$ and normal saline $(2 \mathrm{~mL})$ accompanied by venous occlusion. Induction with microemulsion propofol (Aquafol) $2 \mathrm{mg} / \mathrm{kg}$ was accomplished following the release of venous occlusion. Pain intensity was assessed on a four-point scale according to patient movement (grade 0 , no movement; grade 1, movement in wrist only; grade 2, movement in the upper arm \& shoulder of injected arm; grade 3, generalized movement). Systolic blood pressure, diastolic blood pressure, and heart rate were evaluated.

Results: A significant difference in pain intensity following injection of microemulsion propofol between the groups was found $(P<0.05)$. In addition, the incidence of hypertension after injection was lower in the LM group than in the $L$ group $(P<0.05)$.

Conclusion: The combination of magnesium and lidocaine are effective in attenuating the pain induced by microemulsion propofol injection when compared with lidocaine alone.
\end{abstract}

Keywords: Aquafol; Injections; Lidocaine; Magnesium; Pain; Propofol

\section{INTRODUCTION}

Propofol is a widely used intravenously anesthetic for induction and maintenance of general anesthesia due to its rapid onset and short duration of action. However, previous study found that commonly used long-chain triglyceride formulations (Diprivan) are associated with pain or discomfort following injection in approximately $70 \%$ of patients [1]. Incidence of injection pain mediumchain triglyceride formulations was lower [2], but was still high.

To reduce injection pain, a variety of drugs and non-drug pretreatment methods have been used [3-6]. Lidocaine is commonly used, but cannot completely prevent injection-related pain. Magnesium has been used to prevent the pain caused by propofol injec- tion due its effects as a calcium channel blocker and a physiological antagonist of the N-methyl-D-aspartate (NMDA) receptor $[7,8]$.

Propofol are oils at room temperature and are insoluble in aqueous solution, but are highly lipid soluble. This allows their availability for intravenous administration as an oil-in-water emulsion containing soybean oil, glycerol, and egg lecithin. Propofol formulations can support the growth of bacteria and have been associated with cases of lipidemia, metabolic acidosis, and fat embolism. To address these issues, a hydrosoluble lipid-free microemulsion propofol (Aquafol; Daewon Pharmaceutical Co. Ltd., Seoul, Korea) has been developed and is commercially available $[9,10]$. However, microemulsion propofol injection is known to enhance pain [11]. Data regarding the prevention of this pain are sparse due to its 
short duration of clinical use; indeed, the effectiveness of magnesium sulfate for the pretreatment of microemulsion propofol injection pain has not to our knowledge been investigated. We therefore assessed whether pretreatment with lidocaine and magnesium sulfate prevented the pain associated with IV injection of microemulsion propofol.

This was a randomized, double-blind study of the efficacy of magnesium with lidocaine for the prevention of microemulsion propofol associated injection pain during the induction of general anesthesia. We hypothesized that pretreatment with a combination of magnesium and lidocaine would be equally or more effective than lidocaine alone in terms of reducing the pain associated with injection of microemulsion propofol.

\section{MATERIALS AND METHODS}

Following approval by the institution ethics committee and after obtaining written informed consent from patients, 114 American Society of Anesthesiologists physical status classes I and II patients, 20-70 years of age, and undergoing general anesthesia for surgery were enrolled. Patients were excluded if they had any sign or history indicating existing or previous cardiovascular, hepatic, renal, gastrointestinal and neurological disorders, pregnancy, or allergy to magnesium sulfate.

The patients were assigned randomly according to a computergenerated random table to one of two groups. Group L was pretreated with lidocaine $40 \mathrm{mg}$ and normal saline $2 \mathrm{~mL}(\mathrm{n}=57)$, while group LM was pretreated with a combination of lidocaine $40 \mathrm{mg}$ and magnesium sulfate $10 \mathrm{mg} / \mathrm{kg}(\mathrm{n}=57)$ accompanied by venous occlusion for 1 minute before injection of microemulsion propofol. The patients were premedicated with IM midazolam 2 mg and glycopyrrolate $0.2 \mathrm{mg} 30$ minutes prior to surgery. Before the induction of anesthesia, standard monitoring of heart rate, non-invasive arterial pressure, electrocardiography and arterial oxygen saturation, and bispectral index score were performed. All patients had an 18-gauge IV catheter in a large vein of the upper or lower limbs for the IV line and all drugs were administered near the IV catheter. Anesthesia was induced using microemulsion propofol $(2 \mathrm{mg} / \mathrm{kg})$.

The intensity of pain were assessed during injection of the study microemulsion propofol dose by an anesthesiologist to whom the patient was assigned. Based on Memis et al. [12]' study, we used a four-point scale, in which pain was graded as grade 0 (no pain), grade 1 (mild pain; pain reported in response to questioning and without any behavioral signs), grade 2 (moderate pain; pain reported in response to questioning and accompanied by a behavioral sign, or pain reported spontaneously without questioning), and grade 3 (severe pain; strong vocal response or response accompanied by facial grimacing, arm withdrawal, or tears). Within 6 hours after the operation, symptoms of hypermagnesemia was checked for hypotension, somnolence or respiratory paralysis by an anesthesiologist who was blinded to the patient's group assignment.

Following microemulsion propofol injection and loss of consciousness, rocuronium $0.6 \mathrm{mg} / \mathrm{kg}$ was administered to facilitate controlled ventilation and $6 \mathrm{~L} / \mathrm{min}$ oxygen with sevoflurane (or desflurane) was administered during ventilation via a facemask. Two to three minutes after rocuronium injection, the trachea was intubated and anesthesia was maintained with sevoflurane (or desflurane) and $\mathrm{N}_{2} \mathrm{O} 50 \%$ in oxygen. To maintain systolic blood pressure (SBP) within $30 \%$ of the baseline and a heart rate (HR) under 50/min, patients were administered an intravenous ephedrine, or atropine bolus.

Non-invasive SBP, diastolic blood pressure (DBP), and HR were recorded at baseline, immediately following microemulsion propofol injection and following intubation. The primary endpoint of this study was the pain intensity following microemulsion propofol injection in combination with magnesium. The secondary endpoints were hemodynamic variance and the incidences of hypertension, hypotension, and bradycardia, as required.

Based on previous studies of propofol pain after pretreatment with lidocaine, 50 subjects per group were assigned. The incidence of moderate-to-severe pain following lidocaine pretreatment before injection of microemulsion propofol or lipid soluble was hypothesized to be $50 \%$. After administration of the test drug, a $30 \%$ reduction in moderate-to-severe pain on injection was considered clinically significant. Using $\alpha=0.05$ with a power of $80 \%$, the minimum sample size was predetermined as 51 patients per group and 57 patients per group were allocated after taking into consideration a dropout rate of $10 \%$. Statistical analysis was performed on SPSS ver. 12.0 (SPSS Inc., Chicago, IL, USA). A P-value < 0.05 was considered to indicate significance. Chi-square test was used for categorical data and Student t-test was used of continuous data.

\section{RESULTS}

Of the 114 patients enrolled, all completed the study. No differ- 
Lee SJ, et al. • Magnesium for Injection Pain Prevention

Table 1. Demographic data

\begin{tabular}{lcc}
\hline Variable & Group L (n=57) & Group LM (n=57) \\
\hline Age (yr) & $45 \pm 14$ & $46 \pm 15$ \\
Body weight (kg) & $63 \pm 9$ & $63 \pm 11$ \\
Gender (male/female) & $21 / 36$ & $22 / 35$ \\
$\begin{array}{l}\text { American Society of Anesthesiologists } \\
\text { physical status class (I/II) }\end{array}$ & $39 / 18$ & $40 / 17$ \\
\hline
\end{tabular}

Values are presented as mean \pm standard deviation or number of patients. Group $\mathrm{L}$, $2 \%$ lidocaine $40 \mathrm{mg}$; group LM, 2\% lidocaine $40 \mathrm{mg} \&$ magnesium sulfate $10 \mathrm{mg} /$ $\mathrm{kg}$. No significant differences between the two groups were observed.

Table 2. Pain intensity distributions on the four-point scale ${ }^{\mathrm{a})}$

\begin{tabular}{lcc}
\hline Variable & Group L $(n=57)$ & Group LM $(n=57)$ \\
\hline Grade 0 & 8 & 14 \\
Grade 1 & 23 & 32 \\
Grade 2 & 20 & 9 \\
Grade 3 & 6 & 2 \\
\hline
\end{tabular}

Values are presented as number of patients. Group L, $2 \%$ lidocaine $40 \mathrm{mg}$; group LM, $2 \%$ lidocaine $40 \mathrm{mg} \&$ magnesium sulfate $10 \mathrm{mg} / \mathrm{kg}$.

alThe distribution of scores differed significantly between the groups.

ences in demographic characteristics between the two groups with respect to age, weight, and sex were found (Table 1). The overall pain intensity during microemulsion propofol injection is shown in Table 2. Pain intensity was lower in the LM group than in the $\mathrm{L}$ group $(\mathrm{P}<0.05)$ (Table 2). No differences in SBP, DBP, and HR were observed between the two groups before induction, after aquafol injection, or after intubation. The mean baseline SBP, DBP, and HR values were comparable among the groups (Table 3).

The SBP and DBP values following anesthetic induction before intubation were significantly lower compared to baseline values within the groups $(\mathrm{P}<0.01$ in each group). The SBP and HR values following intubation were significantly higher compared to after aquafol injection within the groups ( $\mathrm{P}<0.001$ in each group). DBP did not differ compared to baseline values within the groups. Intubation caused a significant increase in HR compared to the baseline values in all groups. The incidences of hypertension, hypotension, and bradycardia are shown Table 4. Ephedrine was administered to seven patients in group $\mathrm{L}$ and five patients in group LM due to hypotension. No symptoms of hypermagnesemia or adverse effects were reported.

\section{DISCUSSION}

We demonstrate that the combination of $2 \%$ lidocaine $(40 \mathrm{mg}$ ) and a small dose $(10 \mathrm{mg} / \mathrm{kg})$ of magnesium sulfate were effective
Table 3. Hemodynamic data

\begin{tabular}{llcc}
\hline Variable & \multicolumn{1}{c}{ Category } & $\begin{array}{c}\text { Group L } \\
(\mathrm{n}=57)\end{array}$ & $\begin{array}{c}\text { Group LM } \\
(\mathrm{n}=57)\end{array}$ \\
\hline Systolic blood pressure $(\mathrm{mm} \mathrm{Hg})$ & Baseline & $135 \pm 24$ & $136 \pm 26$ \\
& After aquafol injection & $128 \pm 22^{\text {a) }}$ & $123 \pm 26^{\text {a) }}$ \\
& After intubation & $147 \pm 25^{\text {a) }}$ & $149 \pm 35^{\text {a) }}$ \\
Diastolic blood pressure $(\mathrm{mm} \mathrm{Hg})$ & Baseline & $81 \pm 10$ & $78 \pm 12$ \\
\cline { 2 - 4 } & After aquafol injection & $79 \pm 10$ & $73 \pm 15^{\text {a) }}$ \\
Heart rate (beats/min) & After intubation & $95 \pm 18^{\text {a) }}$ & $92 \pm 23^{\text {a) }}$ \\
& Baseline & $72 \pm 13$ & $69 \pm 14$ \\
& After aquafol injection & $76 \pm 13$ & $76 \pm 13^{\text {a) }}$ \\
& After intubation & $97 \pm 14^{\text {al }}$ & $94 \pm 17^{\text {al }}$ \\
\hline
\end{tabular}

Values are presented as mean \pm standard deviation.

${ }^{a} \mathrm{P}<0.05$ compared to baseline. No significant differences were found between the two groups.

Table 4. Incidences of hypertension, hypotension, and bradycardia

\begin{tabular}{lcc}
\hline Variable & Group L $(\mathrm{n}=57)$ & Group LM $(\mathrm{n}=57)$ \\
\hline Hypertension & 7 & $1^{\text {a) }}$ \\
Hypotension & 7 & 5 \\
Bradycardia & 3 & 0 \\
Grade 3 & 6 & 2 \\
\hline
\end{tabular}

Values are presented as the number of patients. Group L, 2\% lidocaine 40 mg; group LM, 2\% lidocaine $40 \mathrm{mg} \&$ magnesium sulfate $10 \mathrm{mg} / \mathrm{kg}$.

alP $<0.05$ compared with group $\mathrm{L}$.

in reducing microemulsion propofol associated injection pain. In comparison to lidocaine alone, no harmful hemodynamic effects during induction were observed. Approximately $70 \%$ of control patients had pain or discomfort during propofol injection [1], and all patients in each trial reported pain. A variety of drugs and nondrug pretreatments have been used to reduce injection pain. Kim et al. [13] reported that increasing lidocaine dosage significantly reduced pain during injection of microemulsion propofol. Jeong et al. [14] reported that a combination of remifentanil with a premixture of lidocaine was more effective in reducing the incidence of pain upon injection of microemulsion propofol than was a premixture of lidocaine with microemulsion propofol alone.

The microemulsion propofol was developed to address the adverse effects of lipid emulsion propofol, but pain incidence and severity were found to increase. Jung et al. [9] reported that the incidences of pain upon microemulsion and lipid emulsion propofol injection were $81.9 \%$ and $29.2 \%$, respectively. The median visual analog scale score (range, 25\% to 75\%) for injection pain was significantly different between microemulsion (72) and long chain triglycerides (11.5) propofol. The exact cause of the increased incidence and severity of pain is unknown, but a sevenfold higher free 
propofol concentration and activation of the enzymatic cascade of the plasma kallikrein-kinin system is thought to contribute to this effect [11]. In addition, direct stimulation of free nerve endings and A-delta myelinated nociceptors by free propofol has been reported when injection pain occurs. Thus, complete elimination of microemulsion propofol injection pain is problematic.

As a pretreatment agent, lidocaine alone or in combination with other drugs is most commonly used clinically $[11,13,14]$. Lidocaine is an acidic substance, meaning its pretreatment decreases the $\mathrm{pH}$ of propofol, which in turn decreases the aqueous free propofol concentration [11]. The prevention of propofol injection pain is therefore considered to be associated with a reduced free propofol concentration. For this reason, the effects of lidocaine in combination with propofol are greater than pretreatment with lidocaine alone in terms of a reduction in propofol injection pain. Lidocaine has adverse effects, including cardiovascular, neurotoxic and other complications, but the side effects are dose-dependent and the drug is safe at concentrations less than $1 \mathrm{mg} / \mathrm{kg}$. According to recent reports, we administered $40 \mathrm{mg}$ lidocaine [13].

Magnesium is a well-known adjuvant with analgesic properties and anti-arrhythmic effects. It is a natural physiological calcium antagonist and regulates calcium influx into cells.

The analgesic action of some calcium channel blockers could be mediated by an increase of the nociceptive threshold resulting from interference with calcium influx, because the latter is important for the release of neurotransmitters and other substances implicated in nociceptive pain and inflammation [12].

NMDA receptors play an important role in the pain pathway. Magnesium sulfate as a physiological NMDA antagonist is used to reduced intraoperative and postoperative analgesic requirements [7,15]. Magnesium induces direct vasodilation, which is mediated by endothelium-derived nitric oxide and may be responsible for hypotension and tachycardia [12]. Nitric oxide donors protect the vascular endothelium from ischemia or reperfusion. This is a possible mechanism by which magnesium reduces propofol-associated injection pain.

Magnesium sulfate has been shown to enhance the activity of local anesthetic agents. Agarwal et al. [16] reported that magnesium pretreatment causes pain. Based on this study, the authors argued that magnesium was not an appropriate pretreatment for propofolinjection-associated pain. So we did not use magnesium sulfate alone. In our study, we provide evidence that a combination of lidocaine and magnesium is more effective than administration of li- docaine alone.

This study had a number of limitations. Premedication with IM midazolam (2 mg) used just for reduction of anxiety, not for induction or sedation. So, four point scale may not be less objective indicator than verbal rating scale. Also, we did not assess the blood magnesium concentration and were therefore unable to correlate the clinical outcome with the magnesium concentrations in serum and cerebrospinal fluid, but no symptoms of hypermagnesemia (such as hypotension, somnolence, or respiratory paralysis) or adverse effects were reported in our study. Administration of a magnesium sulfate $10 \mathrm{mg} / \mathrm{kg}$ bolus is thought to be safe based on our data. We demonstrate here a significant reduction in pain intensity due to magnesium. The combination of magnesium and lidocaine are effective in attenuating the pain induced by microemulsion propofol injection when compared with lidocaine alone.

\section{REFERENCES}

1. Picard P, Tramer MR. Prevention of pain on injection with propofol: a quantitative systematic review. Anesth Analg 2000;90:963-9.

2. Bachmann-Mennenga B, Ohlmer A, Boedeker RH, Mann M, Muhlenbruch B, Heesen M. Preventing pain during injection of propofol: effects of a new emulsion with lidocaine addition. Eur J Anaesthesiol 2007;24: 33-8.

3. Cheong MA, Kim KS, Choi WJ. Ephedrine reduces the pain from propofol injection. Anesth Analg 2002;95:1293-6.

4. King SY, Davis FM, Wells JE, Murchison DJ, Pryor PJ. Lidocaine for the prevention of pain due to injection of propofol. Anesth Analg 1992;74: 246-9.

5. McCrirrick A, Hunter S. Pain on injection of propofol: the effect of injectate temperature. Anaesthesia 1990;45:443-4.

6. Scott RP, Saunders DA, Norman J. Propofol: clinical strategies for preventing the pain of injection. Anaesthesia 1988;43:492-4.

7. Koinig H, Wallner T, Marhofer P, Andel H, Horauf K, Mayer N. Magnesium sulfate reduces intra- and postoperative analgesic requirements. Anesth Analg 1998;87:206-10.

8. Tramer MR, Schneider J, Marti RA, Rifat K. Role of magnesium sulfate in postoperative analgesia. Anesthesiology 1996;84:340-7.

9. Jung JA, Choi BM, Cho SH, Choe SM, Ghim JL, Lee HM, et al. Effectiveness, safety, and pharmacokinetic and pharmacodynamic characteristics of microemulsion propofol in patients undergoing elective surgery under total intravenous anaesthesia. Br J Anaesth 2010;104:563-76.

10. Lee EH, Lee SH, Park DY, Ki KH, Lee EK, Lee DH, et al. Physicochemical properties, pharmacokinetics, and pharmacodynamics of a reformulated microemulsion propofol in rats. Anesthesiology 2008;109:436-47.

11. Sim JY, Lee SH, Park DY, Jung JA, Ki KH, Lee DH, et al. Pain on injection with microemulsion propofol. Br J Clin Pharmacol 2009;67:316-25.

12. Memis D, Turan A, Karamanlioglu B, Sut N, Pamukcu Z. The use of magnesium sulfate to prevent pain on injection of propofol. Anesth Analg 2002;95:606-8.

13. Kim HS, Cho KR, Lee JH, Kim YH, Lim SH, Lee KM, et al. Prevention of pain during injection of microemulsion propofol: application of lido- 
caine mixture and the optimal dose of lidocaine. Korean J Anesthesiol 2010;59:310-3.

14. Jeong CW, Lee SH, Ju J, Jeong SW, Lee HG. The effect of priming injection of different doses of remifentanil on injection pain of microemulsion propofol premixed with lidocaine. Korean J Anesthesiol 2011;60:78-82.

15. Lysakowski C, Dumont L, Czarnetzki C, Tramer MR. Magnesium as an adjuvant to postoperative analgesia: a systematic review of randomized trials. Anesth Analg 2007;104:1532-9.

16. Agarwal A, Dhiraj S, Raza M, Pandey R, Pandey CK, Singh PK, et al. Vein pretreatment with magnesium sulfate to prevent pain on injection of propofol is not justified. Can J Anaesth 2004;51:130-3. 